\title{
LÓGICA DIFUSA COMO HERRAMIENTA PARA INTERPRETAR DATOS DE PRODUCCIÓN LIMPIA EN EL SECTOR AGRÍCOLA
}

\author{
DIFFUSED LOGIC AS A TOOL FOR INTERPRETING CLEAN \\ PRODUCTION DATA IN THE AGRICULTURE AREA
}

Manuel Rodríguez Molina ${ }^{1}$

\begin{abstract}
RESUMEN
El propósito del presente artículo es dar a conocer principios básicos de lógica difusa en la interpretación de datos en el sector agrícola. Se muestra el caso hipotético de una empresa agrícola a la cual se le realiza una auditoría y posteriormente los datos se analizan bajo el enfoque de lógica difusa con el fin de caracterizar el estado de eficiencia en producción limpia de dicha empresa.

Palabras clave: Lógica difusa, producción limpia, sector agrícola, auditoría, empresa.
\end{abstract}

\section{ABSTRACT}

The purpose of this article is to inform about the basic principles of diffused logic in the interpretation of data obtained in the agriculture area. It shows the hypothetical case of an agriculture enterprise that goes through an audit and whose data are analized afterwards under the concept of a diffused logic with the aim of characterizing the efficiency in the clean production of the enterprise.

Key words: Diffused logic, clean production, agriculture area, audit, enterprise.

\section{INTRODUCCIÓN}

La lógica difusa es concebida en la década del 60 por el ingeniero eléctrico iraní Lofty Zadeh, en la Universidad de California, Berkeley, publicando un artículo titulado "Fuzzy Sets". Posteriormente esta tecnología que nace en EE.UU. es perfeccionada en Europa, y finalmente aplicada en Oriente, principalmente en Japón.

Por otra parte, la producción limpia en la agricultura ya no es una opción, sino más bien un imperativo de sobrevivencia en los mercados altamente competitivos y con significativos estándares de calidad y elevados parámetros de inocuidad. En este sentido los sistemas de análisis, sobre todo de datos, deben ser sensibles y generadores de información fidedigna, que reflejen y modelen de la mejor manera posible, acortando la diferencia entre realidad y teoría, la situación en estudio; en donde las técnicas tradicionales se caracterizan principalmente por aportar información que no refleja lo que ocurre realmente y por una baja riqueza de interpretaciones, basándose principalmente en unos pocos parámetros que esconden o encubren datos de gran valor, necesarios al momento de la toma de decisiones que se orienten en la dirección correcta y permitan realizar una gestión satisfactoria.

En este sentido la lógica difusa ha dado muestras de su gran capacidad de modelamiento y de enriquecimiento, además de "rescatar" particularidades en cada entorno de investigación y aplicación, en donde los sistemas cartesianos tradicionales quedan limitados.

Este trabajo a través de un hipotético pero práctico ejemplo hace una inducción a los conceptos básicos de lógica difusa y una aplicación en el sector agrícola, específicamente en la producción limpia, clasificando una empresa en función de su eficiencia

\footnotetext{
1 Departamento Recursos Ambientales, Universidad de Tarapacá, E-mail: srmanuelrodriguez@yahoo.es, Arica-Chile.
} 
que depende del uso racional y sustentable de agua y agroquímicos, ambos insumos de entrada en el proceso productivo.

\section{Aplicación}

Para ilustrar el proceso de fusificacion, veamos lo que sucedería en una virtual situación de clasificación de una empresa del sector agrícola en función de su desempeño en producción limpia, situación que tendría como criterios norma: eficiencia hídrica y uso de plaguicidas.

Es decir, podríamos seguir el siguiente camino:

\section{$1^{\circ}$. Definición del problema:}

"Clasificar una empresa agrícola en función de su desempeño en producción limpia".

$2^{\circ}$. Definición de un conjunto $\mathrm{X}=\left(\mathrm{x}_{1}, \mathrm{x}_{2}, \mathrm{x}_{3}, \ldots \ldots \ldots \mathrm{x}_{\mathrm{n}}\right)$ de criterios a considerar. En nuestro caso se define:

$\mathrm{x}_{1}=$ cantidad de agua ocupada en la producción de tomate $\left(\mathrm{m}^{3} / \mathrm{ha}\right)$

$\mathrm{x}_{2}=$ cantidad de pesticida ocupado en la producción de tomate $(\mathrm{Ug} / \mathrm{l})$

$3^{\text {o. }}$ Asignar un peso o grado de importancia a cada uno de los criterios, el cual puede ser generado a partir de: estadísticas, panel de expertos. Es decir, se asignará, dentro del universo de discurso, una etiqueta por criterio. Por ejemplo, para el criterio consumo de agua y pesticidas, se asignaron las siguientes etiquetas:

\begin{tabular}{|c|c|c|}
\hline Criterio & Etiqueta & Dominio \\
\hline \multirow{3}{*}{ Agua $\left(\mathrm{m}^{3}\right)$} & Poca & $\mathrm{m}^{3} \leq 3500$ \\
\cline { 2 - 3 } & Normal & $3300<\mathrm{m}^{3}<3700$ \\
\cline { 2 - 3 } & Mucha & $\mathrm{m}^{3} \geq 3600$ \\
\hline
\end{tabular}

\begin{tabular}{|c|l|c|}
\hline Criterio & Etiqueta & Dominio \\
\hline \multirow{4}{*}{$\begin{array}{c}\text { Plaguicida } \\
(\mathrm{Ug} / \mathrm{l})\end{array}$} & Poco & $\mathrm{Ul} \leq 0,08$ \\
\cline { 2 - 3 } & Medio & $0,05<\mathrm{Ul}<0,8$ \\
\cline { 2 - 3 } & Bastante & $0,21 \leq \mathrm{Ul}<1,5$ \\
\cline { 2 - 3 } & Demasiado & $\mathrm{Ul} \geq 1,1$, \\
\hline
\end{tabular}

\section{Gráficamente:}

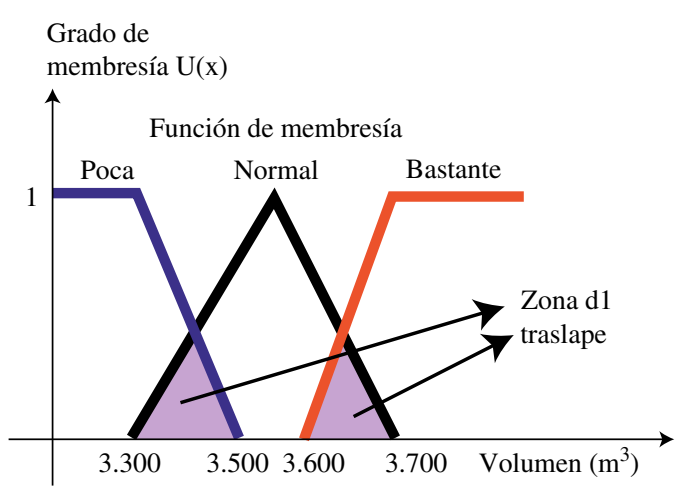

Figura 1. Funciones de membresía para volumen de agua.

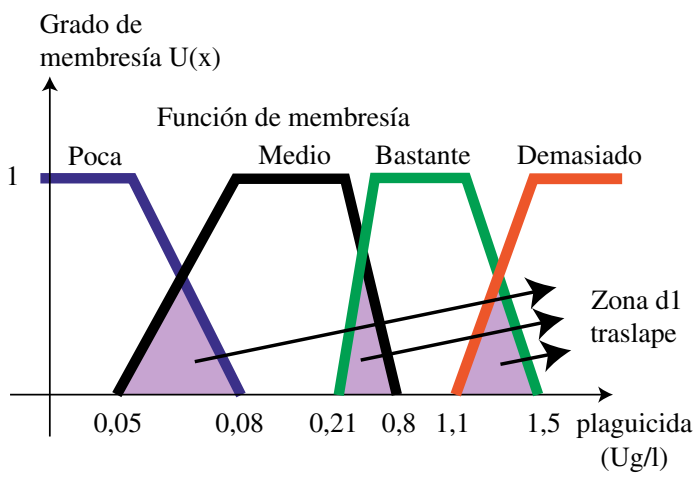

Figura 2. Funciones de membresía para masa de plaguicida.

El eje $\mathrm{U}(\mathrm{x})$ de cada gráfica se refiere al grado de membresía para el cual los valores de entrada pertenecen a la función. Se ha construido una "regla" que permite comparar y clasificar según criterios y pesos definidos.

$4^{\circ}$. Evaluación de una empresa agrícola en cada uno de los atributos (auditoría).

\begin{tabular}{|c|c|}
\hline Agua $\left(\mathbf{m}^{\mathbf{3}}\right)$ & Plaguicida $(\mathbf{U g} / \mathbf{l})$ \\
\hline 3400 & 0,07 \\
\hline
\end{tabular}

$5^{\circ}$. Clasificación y evaluación de la empresa.

En este ejemplo, las etiquetas de las variables consumo de agua y pesticida permiten clasificar la empresa agrícola desde el punto de vista de la 
producción limpia con una eficiencia: alta, media o baja.

Gráficamente:

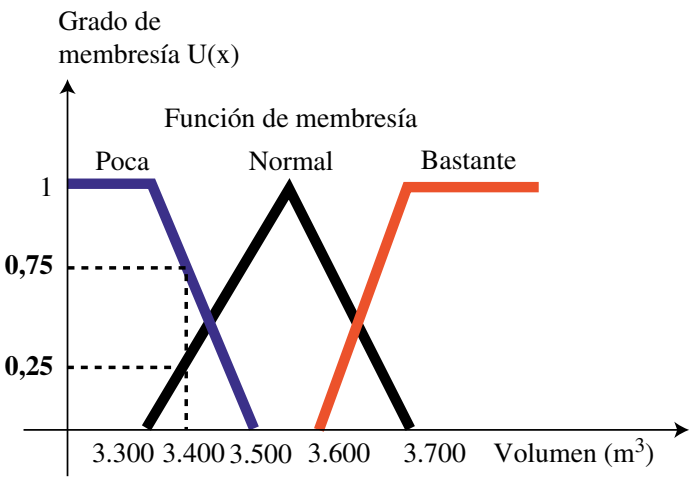

Figura 3. Grado de pertenencia de $V=3400 \mathrm{~m}^{3} \mathrm{t}$ de agua.

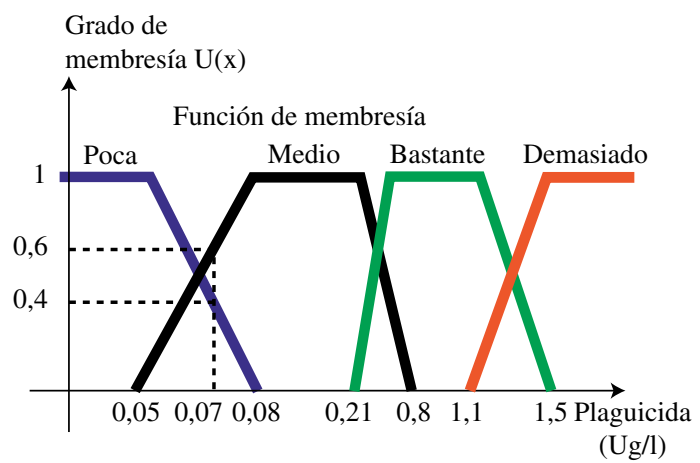

Figura 4. Grado de pertenencia de plaguicida para $C=00,8$ $\mathrm{Ug} / \mathrm{l}$.

Según la matriz, existen 12 potenciales reglas, pero solamente serán ocupadas las reglas que fueron obtenidas por la empresa agrícola después de la auditoría de eficiencia en producción limpia, entonces:

Matriz Agua/Plaguicida

\begin{tabular}{|l|l|l|c|}
\hline Poca & Normal & Bastante & Agua/Plaguicida \\
\hline Alta & Alta & Baja & Poco \\
\hline Media & Media & Media & Medio \\
\hline Baja & Baja & Baja & Bastante \\
\hline Baja & Baja & Baja & Demasiado \\
\hline
\end{tabular}

En el caso en estudio, solamente:

\begin{tabular}{|c|l|}
\hline Regla & \multicolumn{1}{|c|}{ Sentencia } \\
\hline 1 & $\begin{array}{l}\text { Si el consumo de agua es normal y el consumo de } \\
\text { plaguicida es poco la empresa es de eficiencia alta. }\end{array}$ \\
\hline 2 & $\begin{array}{l}\text { Si el consumo de agua es poca y el consumo de } \\
\text { plaguicida es medio la empresa es de eficiencia } \\
\text { media. }\end{array}$ \\
\hline 3 & $\begin{array}{l}\text { Si el consumo de agua es normal y el consumo de } \\
\text { plaguicida es medio la empresa es de eficiencia } \\
\text { media. }\end{array}$ \\
\hline 4 & $\begin{array}{l}\text { Si el consumo de agua es poca y el consumo de } \\
\text { plaguicida es poco la empresa es de eficiencia } \\
\text { alta. }\end{array}$ \\
\hline
\end{tabular}

Los antecedentes poseen un grado de pertenencia, que representan el nivel de veracidad en relación al universo de discurso para cada criterio seleccionado, en donde la regla de fuerza asume como resultado el mínimo valor de los antecedentes, es decir:

\begin{tabular}{|c|l|c|}
\hline Regla & \multicolumn{1}{|c|}{ Sentencia } & min \\
\hline 1 & $\begin{array}{l}\text { Si el consumo de agua es normal }(0,25) \text { y el } \\
\text { consumo de plaguicida es poco }(0,4) \text { la empresa } \\
\text { es de eficiencia alta. }\end{array}$ & 0,25 \\
\hline 2 & $\begin{array}{l}\text { Si el consumo de agua es poca }(0,75) \text { y el } \\
\text { consumo de plaguicida es medio }(0,6) \text { la } \\
\text { empresa es de eficiencia media. }\end{array}$ & 0,6 \\
\hline 3 & $\begin{array}{l}\text { Si el consumo de agua es normal }(0,25) \text { y } \\
\text { el consumo de plaguicida es medio }(0,6) \text { la } \\
\text { empresa es de eficiencia media. }\end{array}$ & 0,25 \\
\hline 4 & $\begin{array}{l}\text { Si el consumo de agua es poca }(0,75) \text { y el } \\
\text { consumo de plaguicida es poco }(0,4) \text { la empresa } \\
\text { es de eficiencia alta. }\end{array}$ & 0,4 \\
\hline
\end{tabular}

Hemos evaluado 4 reglas posibles, ahora se hace necesario comparar las reglas que coinciden en arrojar el mismo consecuente, es decir, la misma clasificación de eficiencia, pero de antecedentes con distintos grados de veracidad. Se puede observar que las reglas 1 y 4 arrojan la misma clasificación, empresa de alta eficiencia, en tanto que las reglas 2 y 3 clasifican a la empresa como de eficiencia media, en todos los casos la clasificación final es determinada por la regla de máxima fuerza: es decir, si dos o más reglas 
sentencian lo mismo, la regla más verdadera se impone, entonces:

La eficiencia de la empresa es media, con un grado de verdad $(0,6)$

La eficiencia de la empresa es alta, con un grado de verdad $(0,4)$

El método de regla de evaluación usado es conocido como min-MAX, porque toma el mínimo de los antecedentes para determinar la fuerza de cada regla y toma la regla más fuerte para cada consecuente, siendo esta la salida. Entonces clasificamos nuestra empresa agrícola como de eficiencia media con un $60 \%$ de certidumbre y como de alta eficiencia con un $40 \%$ de certeza. Concluyendo, la empresa obtiene el siguiente gradiente de veracidad para cada una de las etiquetas asignadas en cada caso:

La eficiencia de la empresa es baja, con un grado de verdad (0)

La eficiencia de la empresa es media, con un grado de verdad $(0,6)$

La eficiencia de la empresa es alta, con un grado de verdad $(0,4)$

\section{CONCLUSIÓN}

Por medio de la lógica difusa se puede caracterizar una empresa agrícola con diferentes grados de eficiencia en función del manejo de los recursos de que dispone, pero a diferencia de un sistema tradicional de comparación con una norma preestablecida, en cuyo caso la clasificación hubiera sido en función de un parámetro rígido (por ejemplo consumo no mayor a $3.700 \mathrm{~m}^{3}$ de agua es eficiente, en otro caso es ineficiente), la lógica difusa aporta y enriquece con más información porque realiza la clasificación y asigna una ponderación a ésta, pero además también se pueden crear otros escenarios si se siguen tomando hacia atrás las clasificaciones que precedieron a la de mayor puntuación: en nuestro ejemplo, la empresa en cuestión también es de eficiencia alta con un grado de verdad 0,25, que es una de las clasificaciones desplazadas al comparar las mismas salidas para un mismo criterio, pero con distinta ponderación final, esto permite tener más de un escenario al momento de escoger estrategias de optimización y búsqueda de soluciones. Por otra parte, este tipo de abordaje hace posible el "rescate" de la opinión de expertos, como puede ser un trabajador agrícola, al momento de definir las etiquetas de las funciones de pertenencia; también logra caracterizar dichos criterios en el universo de discusión en cuestión, por ejemplo, la eficiencia hídrica puede ser más crítica (mayor peso del criterio en la evaluación) en la agricultura del norte del país que en el sur, es decir, se pueden contemplar particularidades y situaciones singulares que permitan pasar de información cuantitativa a una de tipo cualitativo, logrando así mejores herramientas para la toma de decisiones. También es pertinente indicar que si bien el presente informe trabaja con una sola empresa hipotética, es perfectamente extensible a un mayor número de ellas, lo cual permitiría realizar comparaciones y clasificarlas; análogamente podemos decir lo mismo de los criterios ya que el enfoque de la lógica difusa permite analizar a la diferencia de los sistemas tradicionales de manipulación de datos numéricos, trabajar en forma ascendente de manera multivariable agregando más información sin por ello perder coherencia, abarcando así problemas de alta complejidad multivaluada.

Técnicas como la descrita en este artículo cobran relevancia en el sector agrícola debido a su alta heterogeneidad de modelos de producción, escenarios siempre cambiantes, dependencia de factores difíciles de modelar por los métodos tradicionales (clima), gran incertidumbre de eventos y constante evolución de los mercados que ejercen presión sobre los modos de producción. Esto es de importancia mayor cuando países como Chile pretenden ser potencias agroalimentarias con una economía abierta y permeable a los mercados externos que son exigentes y con elevados estándares de calidad e inocuidad, máxime en el último tiempo en que las variables ambientales llevan a definir criterios paraarancelarios (por ejemplo, cadena de $\mathrm{CO}_{2}$ ) asociados a esta condición (dumping ambiental). 


\section{LITERATURA CONSULTADA}

BARROS, L. C. ET AL. 1998. Epidemiologia e Teoria Fuzzy, Revista de Biomatemática. IMECC-UNICAMP, Volumen IX, 1999.

JORQUERA, H. ET AL. Manual de auditoría en producción limpia. Ministerio de Economía de Chile: Secretaría Ejecutiva de Producción Limpia.

MORENO, E. ET AL. 2006. Perfeccionamiento del modelo ADOMA mediante la inclusión de la ambigüedad en alguno de sus parámetros. Agricultura Técnica Chile, Volumen 66, Número 2.

ROMÁN, H. 1998. Análisis Fuzzy Multívoco. $1^{\text {er }}$ Concurso de creación intelectual, área de investigación, UTA.

TAMAMES, R. 2002. Agricultura de conservación 2002: un enfoque global. Instituto de Cuestiones Agrarias y Medioambientales. Ediciones Mundi prensa, Barcelona. 